\section{Nebivolol: una nueva droga para el tratamiento de la hipertensión arterial}

Los betabloqueantes han tenido durante muchos años un pape preponderante en el tratamiento de la hipertensión arterial, con probada eficacia tanto para controlar las cifras tensionales como para disminuir la morbimortalidad relacionada a esta enfermedad. Ha surgido una nueva clase de bloqueante selectivo de los receptores beta, el Nebivolol, que posee además un mecanismo de acción asociado a la modulación de las vías endoteliales del óxido nítrico.

\section{Perfil farmacológico y farmacodinámico}

El Nebivol es una mezcla racémica de dos enantiómeros I y d nebivolol y actúa ligándose en forma competitiva y altamente selectiva a los receptores beta 1, comportándose como antagonista. Ejerce además propiedades vasodilatadoras dependientes de la dosis, demostradas en el lecho arterial y en el venoso, en presencia de endotelios tanto sanos como enfermos, incluyendo vasos de pacientes hipertensos. Esta vasodilatación, que incluye el aumento del flujo en las arterias coronarias y la disminución de la pre y postcarga, estaría mediada por la síntesis de óxido nítrico estimulada por el nebivolol o alguno de sus metabolitos.

\section{Perfil farmacocinético}

El nebivolol se absorbe en forma rápida por vía oral y no se encuentra influenciado por los alimentos. Alcanza concentraciones pico dentro de los 30 minutos a dos horas luego de su administración. Su vida media es de 2 horas, pero sufre una extensa metabolización hepática, sitio potencial de interacción con otros fármacos como la cimetidina, quinidina, fluoxetina, paroxetina y propafenona. A este nivel se produce un metabolito activo hidroxilado, cuya vida media es de 20 horas. Existe un polimorfismo genético metabólico, aunque en ensayos clínicos no se han encontrado diferencias en los efectos hemodinámicos del nebivolol entre diferentes metabolizadores.

A pesar de que la excreción urinărià de nebivolol es menor al 0,5\% de la dosis, se han detectadô aumentos en la concentración de la droga en pacientes hipertensos con enfermedad renal moderada a severa, por lo que se recomienda comenzar en estos pacientes con $2,5 \mathrm{mg}$ diarios e ir aumentando hasta $5 \mathrm{mg}$ diarios si fuera necesario.

El nebivolol se encuenträ contraindicado en pacientes con insuficiencia hepática.

En relación a las modificaciones de distribución con la edad avanzada, se recomienda comenzar también con la mitad de la dosis en pacientes de más de 65 años y tener en cuenta que el nebivolol parece ligarse al tejido graso, por lo que habría que ajustar la dosis en pacientes obesos.

\section{Eficacia}

En un estudio aleatorizado multicéntrico, doble ciego, con grupos paralelos de 509 pacientes seguidos por cuatro semanas, con hipertensión arterial primaria, el nebivolol a dosis de $2,5,5$ y $10 \mathrm{mg}$ (pero no menores) redujo significativamente la presión arterial diastólica entre 7 a $10,2 \mathrm{~mm} \mathrm{Hg}(p<0,05)$ comparado con placebo. No se registraron diferencias significativas en los grupos de 5 y $10 \mathrm{mg}$.

En un estudio no comparativo de nebivolol $5 \mathrm{mg}$ en una toma diaria en hipertensos esenciales moderados ( TAD entre 94 y 114 $\mathrm{mmHg}$ ) la reducción significativa de la TA se mantuvo durante las 12 semanas de tratamiento $(p<0,001)$.

Otros estudios que compararon nebivolol vs otros antihipertensivos encontraron una reducción similar en la TA a lo largo de 24 horas con 2,5 a $10 \mathrm{mg} /$ día de nebivolol comparado con lisinopril 10 a $40 \mathrm{mg} / \mathrm{d}$ ía, enalapril $10 \mathrm{mg} / \mathrm{día}$, atenolol $100 \mathrm{mg} / \mathrm{día}$ y nifedipina $20 \mathrm{mg}$ dos veces por día en seguimiento de hasta 12 semanas $(p<0,01)$.

En uno de estos estudios hubo un porcentaje mayor de respondedores en el grupo de nebivolol que en el grupo de enalapril, pero las TAD basales eran significativamente mayores en el grupo enalapril y se utilizó la dosis de 10 en lugar de la de $20 \mathrm{mg}$ en este grupo, por lo que no puede afirmarse la mayor eficacia del nebivolol. El nebivolol parece prevenir mejor que la nifedipina los aumentos matinales de TA.

Un metanális que evaluó la eficacia antihipertensiva del nebivolo en dosis de 0,5 a $10 \mathrm{mg}$ día comparando con placebo, atenolol 50 o $100 \mathrm{mg}$ día, metoprolol $100 \mathrm{mg}$ día y otros antihipertensivos como nifedipina $20 \mathrm{mg}$ día, lisinopril $2,5 \mathrm{mg}$ día, enalapril $10 \mathrm{mg}$ día y en combinación con hidroclorotiazida 12,5 o 25 mg día demostró resultados coincidentes: $5 \mathrm{mg}$ de nebivolol una vez por día redujeron significativamente la TA promedio, y esta reducción se mantuvo durante la teräpéutica continuada, sin HTA de rebote o tolerancia. Fue igualmente efectiva que el resto de los antihipertensivos utilizados para comparar y posee un efecto aditivo al combinarla con hidroclorotiazida $12,5 \mathrm{mg}$ día pero no con enalapril.

\section{Efectos adversos y seguridad}

El nebivolol ha sido estudiado en más de 3000 pacientes hipertensos, que recibieron la droga desde al menos un mes hasta tres años.

Los efectos adversos más frecuentes (1 a $10 \%$ ) fueron cefalea, mareos, cansancio y parestesias, todos transitorios y de intensidad leve. No se observó hipotensión ortostática en ninguno de los trabajos:

El número de pacientes que presentaron disfunción sexual erectil o disminución de la libido fue mayor en los grupos atenolol y enalapril que en el nebivolol.

Los ensayos clínicos han mostrado una baja frecuencia de efectos adversos y que no han alterado la calidad de vida de los pacientes. En relación a los efectos metabólicos del nebivolol, no se han de tectado efectos deletéreos en el metabolismo de la glucosa o lípidos en päciente hipertensos, aunque ha habido reportes aislados de elevación de triglicéridos sanguíneos.

\section{Contraindicaciones}

Son las mismas que se aplican a los beta bloqueantes en general. Se encuentra contraindicado en el embarazo, lactancia y enfermedad hepática.

\section{Dosis en la hipertensión arterial}

La dosis habitual es de $5 \mathrm{mg}$ en una toma diaria, preferentemente a la misma hora. Puede administarse con alimentos. El efecto sobre la presión arterial se observa entre las dos a cuatro semanas de comenzado èl tratamiento.

Se presentä en comprimidos de $5 \mathrm{mg}$, su nombre comercial en Argentina es NEBILET por 28 comprimidos, y el costo del envase de $\$ 67$ 


\section{Comentario}

El nebivolol ha demostrado ser tan eficaz como otros antihipertensivos actualmente en uso para reducir los niveles tensionales en pacientes con hipertensión arterial leve a moderada, por lo que algunos autores lo recomiendan como primera línea de tratamiento.

Parece una droga segura, aunque no existen estudios de seguimiento de más de tres años.

Debido a su farmacodinamia ligada al óxido nítrico y que ha demostrado provocar vasodilatación aun en endotelios disfuncionales, se ha postulado que sería más efectiva que otros antihipertensivos en pacientes diabéticos o dislipémicos, en los cuales el endotelio dañado jugaría un papel esencial, pero hasta el momento no se ha probado en ensayos clínicos.

El nebivolol parece carecer de efecto inotrópico negativo aun en pacientes con deterioro de función ventricular (se postula incluso un leve efecto inotrópico positivo) por lo que podría estar indicado en pacientes hipertensos con insuficiencia cardíaca congestiva (ICC), aunque faltan estudios que evalúen puntos finales duros, como mortalidad, tanto para su efecto antihipertensivo como para antianginoso y en insuficiencia cardíaca. Recordemos que aunque durante muchos años los beta bloquenates no eran indicados en insuficiencia cardíaca por su inotropismo negativo, hoy se conoce su efecto beneficioso en la morbimortalidad de estos pacientes. Es por ello importante que las hipótesis fisiopatológicas sean evaluadas en ensayos clínicos rigurosos; que muchas veces contradicen lo esperado "fisiopatológicamente".

\section{¿Cuál es la ventaja del nebivolol versus otros beta blo- queantes en el tratamiento de pacientes hipertensos?}

El nebivolol ha sido comparado solamente con atenolol y metoprolol. De estos estudios surge que los pacientes tratados con nebivolol presentaron menos fatiga y levemente mayor tolerancia al ejercicio que con los otros dos beta bloqueantes, con similares resultados antihipertensivos en cuanto a descenso de $\mathrm{mmHg}$ (no a morbimortalidad).

El nebivolol tendría un efecto benéfico sobre la función ventricular izquierda, lo cual en teoría resultaría beneficioso en hipertensos con disfunción ventricular. No existen estudios que hayan comparado los beta bloqueantes claramente beneficiosos en CC (carvedilol, metoprolol, bisoprolol) con el nebivolol en pacientes hipertensos o con ICC, ni estudios que hayan evaluado eficacia secundaria del nebivolol, como secuelas y mortalidad.

Otro punto a evaluar es el costo del tratamiento, en especial en un tratamiento crónico como el de la hipertensión arterial. El costo de un tratamiento con $5 \mathrm{mg}$ diarios de nebivolol es cuatro veces superior al del enalapril $20 \mathrm{mg}$, ocho o más veces superior al tratamiento con tiazidas, nueve veces mayor que el de $50 \mathrm{mg}$ de atenolol y cuesta un tercio más que $25 \mathrm{mg}$ diarios de carvedilol.

A la luz de la evidencia disponible hasta el momento, el nebivolol parece una droga eficaz para el tratamiento de pacientes hipertensos leves a moderados, aunque no se ha evaluado si disminuye la morbimortalidad secundaria a esta enfermedad. Tampoco existe mucha experiencia sobre la seguridad a largo plazo de esta droga y su costo es significativamente mayor que el de otros tratamientos con demostrada eficacia y seguridad. Es por ello que el nebivolol no es por ahora una droga de primera línea de tratamiento antihipertensivo. Restan esperar resultados de ensayos clínicos a largo plazo que evalúen resultados "duros" como mortalidad y eventos vasculares en vez de los "blandos" como reducción de $\mathrm{mmHg}$ para evaluar el lugar potencial de esta droga en el tratamiento antihipertensivo.

Dra. Victoria Wurcel [ Unidad de Medicina Familiar y Preventiva. Hospital Italiano de Buenos Aires ]

\section{Referencias}

1-UK drug information pharmacist group, Nebivolol,August 1999.

2-DARE: Goa et al,Nebivolol in the management of essential hypertension: a review: Drugs 1999; 57(4):633-651

3-Revista Kairos, 2002

4-Van Nueten et al; Nebivolol vs atenolol and placebo in essential hypertension: a double blind randomised trial.Journal of human hypertension (1998), 12 135-140

5-Van Nueten et al; Nebivolol vs enalapril in the treatment of essential hypertension: a double blind randómised trial. Journal of human hypertension(1997) 11.813-819.

6-Van Borter et al:; Nebivolol in hypertension: a double blind placebo-controlled multicenter study assessing its antyhipertensive efficacy and impact on quality of life. Journal of cardiovascular pharmacology. 1993.21;856-862.

7-De cree et al, Comparative cardiac haemodynamics of bisoprolol, celiprolol, carvedilol and nebivolol in normal volunteers:Int.J.Clin. Pharm. Res.XII. (4) 159-163 (1992)

8- Foody JM, Farrell MH, Krumholz HM. beta-Blocker therapy in heart failure: scientific review. JAMA 2002 Feb 20;287(7):883-9

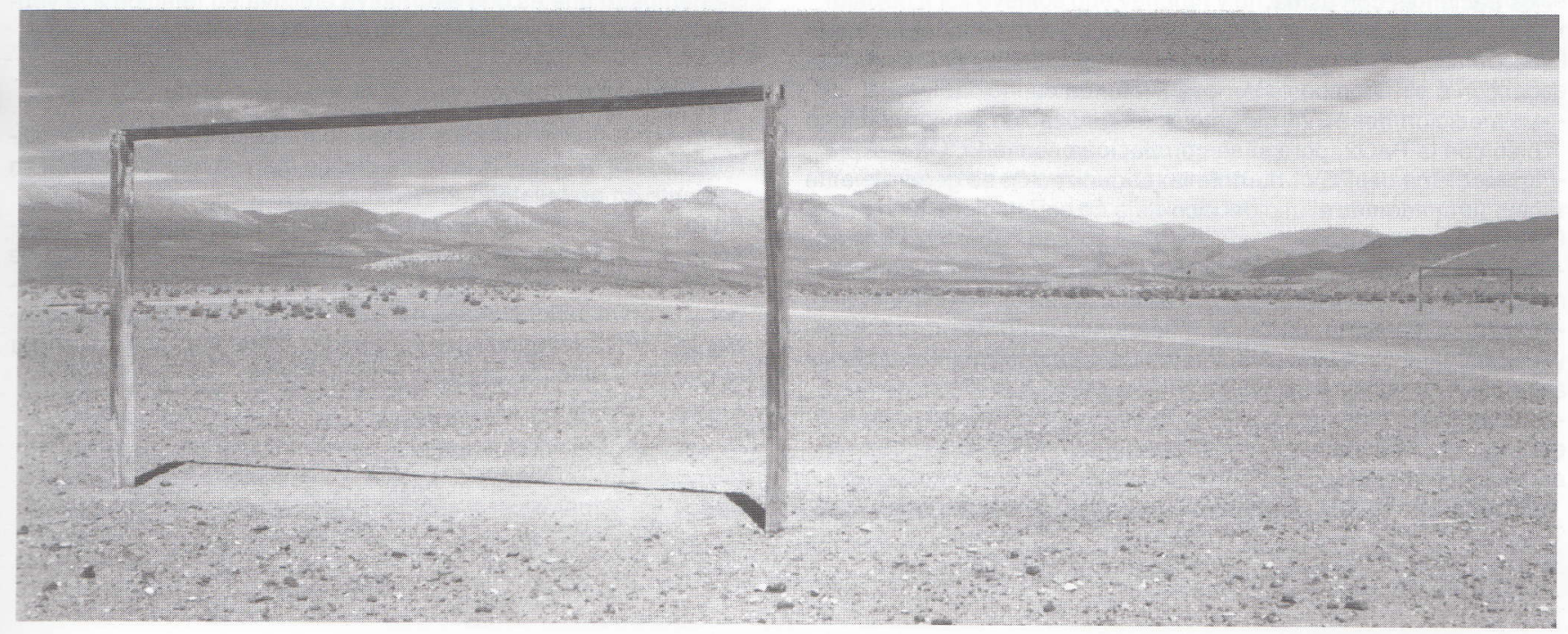

